PROCEEDINGS OF THE

AMERICAN MATHEMATICAL SOCIETY

Volume 129, Number 11, Pages 3353-3362

S 0002-9939(01)05992-5

Article electronically published on April 9, 2001

\title{
BLOWUP IN A MASS-CONSERVING CONVECTION-DIFFUSION EQUATION WITH SUPERQUADRATIC NONLINEARITY
}

\author{
TODD L. FISHER AND CHRISTOPHER P. GRANT
}

(Communicated by David S. Tartakoff)

\begin{abstract}
A nonlinear convection-diffusion equation with boundary conditions that conserve the spatial integral of the solution is considered. Previous results on finite-time blowup of solutions and on decay of solutions to the corresponding Cauchy problem were based on the assumption that the nonlinearity obeyed a power law. In this paper, it is shown that assumptions on the growth rate of the nonlinearity, which take the form of weak superquadraticity and strong superlinearity criteria, are sufficient to imply that a large class of nonnegative solutions blow up in finite time.
\end{abstract}

\section{INTRODUCTION}

Consider the initial-boundary value problem $\mathcal{P}\left(u_{0}, f, T\right)$ :

$$
\begin{cases}u_{t}=u_{x x}+(f(u))_{x}, & (x, t) \in(0,1) \times(0, T), \\ u_{x}=-f(u), & (x, t) \in\{0,1\} \times(0, T), \\ u(x, 0)=u_{0}(x), & x \in[0,1],\end{cases}
$$

where $f \in C^{2}(\mathbb{R}, \mathbb{R}), u_{0} \in C^{2+\beta}([0,1], \mathbb{R})$ for some $\beta>0, u_{0}^{\prime}(x)=-f\left(u_{0}(x)\right)$ for $x \in\{0,1\}$, and $T \in(0, \infty]$. The combination of general existence theory for parabolic equations in divergence form (see, e.g., Chapter V, $\S 7$, of [7]) with a cutoff argument guarantees that, for each such $f$ and $u_{0}$, there exists $T>0$ such that $\mathcal{P}\left(u_{0}, f, T\right)$ has a unique solution $u \in C^{2+\beta, 1+\beta / 2}([0,1] \times[0, T), \mathbb{R})$. Furthermore, $\mathcal{P}\left(u_{0}, f, \infty\right)$ has a solution unless $\mathcal{P}\left(u_{0}, f, T\right)$ has an unbounded solution for some $T<\infty$.

Let

$$
\mathcal{T}\left(u_{0}, f\right):=\sup \left\{T \in(0, \infty] \mid \mathcal{P}\left(u_{0}, f, T\right) \text { has a solution }\right\},
$$

and let $\mathcal{U}\left(u_{0}, f\right)$ be the unique solution of $\mathcal{P}\left(u_{0}, f, \mathcal{T}\left(u_{0}, f\right)\right)$. If $\mathcal{T}\left(u_{0}, f\right)<\infty$, then we say that $\mathcal{U}\left(u_{0}, f\right)$ blows up in finite time or, more specifically, that $\mathcal{U}\left(u_{0}, f\right)$ blows up at time $\mathcal{T}\left(u_{0}, f\right)$. In this paper, we describe some conditions on $f$ and $u_{0}$ that guarantee finite-time blowup of $\mathcal{U}\left(u_{0}, f\right)$.

Since the solution $u$ of this problem is often thought of as the density of some substance, we will only consider nonnegative solutions. We will also confine our attention to unidirectional convection and will assume that convection is zero if and only if density is zero; taking the convection to be leftward, without loss of

Received by the editors March 23, 2000.

1991 Mathematics Subject Classification. Primary 35B30, 35B40, 35K20, 35K60.

(C)2001 American Mathematical Society 
generality, we have $f(0)=0$ and $f((0, \infty)) \subseteq(0, \infty)$. For such $f$, the maximum principle for parabolic equations $[8]$ indicates that $\mathcal{U}\left(u_{0}, f\right)$ will be nonnegative if and only if $u_{0}$ is nonnegative.

When $f(s):=s^{2}, \mathcal{P}\left(u_{0}, f, T\right)$ is a version of Burgers' equation, and with this quadratic nonlinearity no solutions blow up in finite time; on the other hand, if $f(s):=s^{n}$ for some $n>2$, then for some $u_{0}$ the corresponding solution $u=\mathcal{U}\left(u_{0}, f\right)$ undergoes finite-time blowup and, in fact,

$$
\left\{u(0, t) \mid t \in\left[0, \mathcal{T}\left(u_{0}, f\right)\right)\right\}
$$

is unbounded [1]. The corresponding Cauchy problem in one and several space dimensions for these same power-law nonlinearities was studied in [2, 3] [4], [5], and [9], and results relating the asymptotic rate of decay to the size of the exponent were obtained.

Here, we will not restrict $f$ to be given by a particular sort of algebraic formula but will instead impose general growth conditions on $f$.

The total amount at time $t$ of the substance whose density is $u=\mathcal{U}\left(u_{0}, f\right)$ is given by $\int_{0}^{1} u(x, t) d x$. Because of the boundary conditions, this quantity is constant in time and, therefore, is equal to the initial mass $\mathcal{M}\left(u_{0}\right):=\int_{0}^{1} u_{0}(x) d x$. Given $f$, let

$$
\mathcal{E}(f):=\left\{u_{0}:[0,1] \rightarrow[0, \infty) \mid u_{0}^{\prime}=-f\left(u_{0}\right)\right\}
$$

and let

$$
\mathcal{M}_{c}(f):=\sup \left\{\mathcal{M}\left(u_{0}\right) \mid u_{0} \in \mathcal{E}(f)\right\} \in[0, \infty]
$$

We will call $\mathcal{E}(f)$ the set of equilibria, and $\mathcal{M}_{c}(f)$ will be called the critical mass of $f$. It will turn out that if $f$ grows rapidly enough, then $\mathcal{U}\left(u_{0}, f\right)$ will blow up in finite time if $\mathcal{M}\left(u_{0}\right)>\mathcal{M}_{c}(f)$, or if the initial distribution of the substance is highly concentrated near $x=0$.

Let $g:(0, \infty) \rightarrow(0, \infty)$ be defined by the formula $g(s):=f(s) / s$, and consider the following list of hypotheses about $g$ :

(SL1): $g$ is (strictly) increasing;

(SL2): $\liminf _{s \uparrow \infty} s g^{\prime}(s)>0$;

(SL3): for every $\lambda \in(0,1), \liminf _{s \uparrow \infty}[g(s)-g(\lambda s)]>0$;

(SL4): for some $\lambda \in(0,1), \liminf _{s \uparrow \infty}[g(s)-g(\lambda s)]>0$;

(SQ): $\int_{1}^{\infty} \frac{d s}{g(s)}<\infty$.

The label attached to each of these hypotheses signifies that it is either some sort of assumption of superlinear growth of $f,(\mathbf{S L 1}-4)$, or of superquadratic growth of $f,(\mathbf{S Q})$. Note, however, that (SQ) does not imply any of the superlinearity assumptions.

Our first main result, proved in Section 2, is about the blowup of all solutions with sufficiently large mass:

Theorem 1.1. Suppose (SQ), (SL1), and either (SL2) or (SL3) hold. Then $\mathcal{M}_{c}(f)<\infty$, and if $\mathcal{M}\left(u_{0}\right)>\mathcal{M}_{c}(f)$, then $\mathcal{U}\left(u_{0}, f\right)$ blows up in finite time.

Our second main result, proved in Section 3, is about solutions with arbitrarily small mass that blow up in finite time: 
Theorem 1.2. Suppose that (SQ), (SL1), and (SL4) hold. Then for every $\varepsilon>0$, there exists $u_{0}$ such that $\mathcal{M}\left(u_{0}\right)<\varepsilon$ and $\mathcal{U}\left(u_{0}, f\right)$ blows up in finite time.

\section{SuperCRiticAl BlOWup}

Lemma 2.1. If (SQ) holds, then there is a function $\psi:(0, \infty) \rightarrow(0, \infty)$ with the properties:

1. $\lim _{x \downarrow 0} \psi(x)=\infty$;

2. $\psi^{\prime}=-f(\psi)$;

3. $\mathcal{M}_{c}(f)=\mathcal{M}(\psi)<\infty$.

Proof. Define $F:(0, \infty) \rightarrow(0, \infty)$ by the formula

$$
F(\sigma):=\int_{\sigma}^{\infty} \frac{d s}{f(s)}
$$

By (SQ),

$$
\left|\int_{a}^{b} \frac{d s}{f(s)}\right| \leq \frac{1}{\min \{a, b\}}\left|\int_{a}^{b} \frac{d s}{g(s)}\right| \rightarrow 0
$$

as $a, b \uparrow \infty$, so $F$ is well-defined. Note that $F$ is decreasing and

$$
\lim _{\sigma \uparrow \infty} F(\sigma)=0 .
$$

Furthermore, for small positive values of $x, f(x) \leq\left(f^{\prime}(0)+1\right) x$, so $\lim _{y \downarrow} F(y)=\infty$. Hence, $F$ is a bijection; let $\psi:(0, \infty) \rightarrow(0, \infty)$ be the inverse of $F$.

Property 1 of $\psi$ follows from (2.1). Since

$$
\psi^{\prime}(x)=\frac{1}{F^{\prime}(\psi(x))}=-f(\psi(x)),
$$

$\psi$ satisfies Property 2.

For each $u_{0} \in \mathcal{E}(f), u_{0}$ and $\psi$ satisfy the same ordinary differential equation, with $u_{0}(x)<\psi(x)$ for $x$ near 0 , so $u_{0}(x)<\psi(x)$ for every $x \in(0,1]$, by the PicardLindelöf Theorem [6]. This means that $\mathcal{M}\left(u_{0}\right)<\mathcal{M}(\psi)$, so $\mathcal{M}_{c}(f) \leq \mathcal{M}(\psi)$. The substitution $s=\psi(x)$ yields

$$
\mathcal{M}(\psi)=\int_{0}^{1} \psi(x) d x=\int_{\infty}^{\psi(1)} \frac{-s}{f(s)} d s=\int_{\psi(1)}^{\infty} \frac{d s}{g(s)}=\int_{\psi(1)}^{1} \frac{d s}{g(s)}+\int_{1}^{\infty} \frac{d s}{g(s)}<\infty
$$

by (SQ) and the positivity of $g$. To complete the verification that $\psi$ has Property 3 , note that for each $\delta \in(0,1)$

$$
\begin{aligned}
\mathcal{M}(\psi) & =\int_{0}^{\delta} \psi(x) d x+\int_{\delta}^{1} \psi(x) d x \leq \int_{0}^{\delta} \psi(x) d x+\mathcal{M}\left(\psi_{\delta}\right) \\
& \leq \int_{0}^{\delta} \psi(x) d x+\mathcal{M}_{c}(f),
\end{aligned}
$$

where $\psi_{\delta} \in \mathcal{E}(f)$ is the function given by the formula $\psi_{\delta}(x)=\psi(x+\delta)$. Letting $\delta \downarrow 0$ and using the fact that $\mathcal{M}(\psi)<\infty$, we get $\mathcal{M}(\psi) \leq \mathcal{M}_{c}(f)$.

Proof of Theorem 1.1. Suppose that (SQ), (SL1), and either (SL2) or (SL3) hold. Then $\mathcal{M}_{c}(f)<\infty$ by Lemma 2.1 . 
Suppose (SL2) holds, and fix $\lambda \in(0,1)$. By the Mean Value Theorem, for each $s \in(0, \infty)$ there is $\theta \in(\lambda s, s)$ such that

$$
g(s)-g(\lambda s)=(s-\lambda s) g^{\prime}(\theta)=\frac{(1-\lambda) s}{\theta} \theta g^{\prime}(\theta) .
$$

Since $(1-\lambda) s / \theta>(1-\lambda)>0$ and $\liminf _{\theta \uparrow \infty} \theta g^{\prime}(\theta)>0, \liminf _{s \uparrow \infty}[g(s)-g(\lambda s)]>0$, so (SL3) holds.

It is, therefore, sufficient to show that the conditions (SL1) and (SL3) and the inequality $\mathcal{M}\left(u_{0}\right)>\mathcal{M}_{c}(f)$ imply that $\mathcal{U}\left(u_{0}, f\right)$ blows up in finite time. We will do this by assuming, to the contrary, that $\mathcal{T}\left(u_{0}, f\right)=\infty$ and then using an indirect comparison argument to arrive at a contradiction.

Pick $k \in\left(1, \mathcal{M}\left(u_{0}\right) / \mathcal{M}_{c}(f)\right)$, and let $\lambda=1 / k \in(0,1)$. By (SL3),

$$
\liminf _{s \uparrow \infty}[g(s)-g(\lambda s)]>0 .
$$

By (SL1), $g(s)-g(\lambda s)>0$ for every $s \in(0, \infty)$ and, therefore, by the continuity of $g$,

$$
\inf \{g(s)-g(\lambda s) \mid s \in[k \psi(1), b]\}>0
$$

for any $b>k \psi(1)$, where $\psi$ is the function provided by Lemma 2.1 Combining (2.2) and (2.3), we see that we can pick $T \in(0, \infty)$ such that for every $s \geq k \psi(1)$

$$
T>\frac{1}{g(s)-g(\lambda s)} .
$$

Let $\Omega=\{(x, t) \in(0,1) \times(0, T) \mid x>1-t / T\}$. Define $\varphi:[0, \infty) \rightarrow[0, \infty)$ by the formula

$$
\varphi(x)=\int_{0}^{x} \psi(s) d s
$$

and define $z: \bar{\Omega} \rightarrow[0, \infty)$ by the formula $z(x, t)=k \varphi(x+t / T-1)$. Let $u=\mathcal{U}\left(u_{0}, f\right)$, and define $v: \bar{\Omega} \rightarrow[0, \infty)$ by the formula

$$
v(x, t)=\int_{0}^{x} u(s, t) d s .
$$

Define $w: \bar{\Omega} \rightarrow \mathbb{R}$ by the formula $w(x, t)=e^{-t}(z(x, t)-v(x, t))$. Note that $z(0, T)=v(0, T)=0$ and $v_{x}(0, T)=u(0, T)<\infty$, while

$$
\lim _{x \downarrow 0} \frac{z(x, T)-z(0, T)}{x}=k \lim _{x \downarrow 0} \psi(x)=\infty,
$$

so $w(x, T)$ is positive for some $x \in(0,1]$. Since $w$ is continuous on the compact set $\bar{\Omega}$, it attains a positive maximum at some point, say, $\left(x_{0}, t_{0}\right) \in \bar{\Omega}$.

Since $w(x, t)=-e^{-t} v(x, t) \leq 0$ if $x=1-t / T$, and

$$
w(1, t)=e^{-t}\left(k \varphi(t / T)-\int_{0}^{1} u(s, t) d s\right) \leq e^{-t}\left(k \mathcal{M}_{c}(f)-\mathcal{M}\left(u_{0}\right)\right)<0,
$$

either $\left(x_{0}, t_{0}\right)$ is in $(0,1) \times\{T\}$ or in $\Omega$. In either case, calculus implies that

$$
\begin{aligned}
w_{t}\left(x_{0}, t_{0}\right) & \geq 0, \\
w_{x}\left(x_{0}, t_{0}\right) & =0,
\end{aligned}
$$

and

$$
w_{x x}\left(x_{0}, t_{0}\right) \leq 0
$$


Now, from the definition of $v$, we see that

$$
\begin{aligned}
v_{t}(x, t) & =\int_{0}^{x} u_{t}(s, t) d s=\int_{0}^{x}\left[u_{s s}(s, t)+(f(u(s, t)))_{s}\right] d s \\
& =u_{x}(x, t)+f(u(x, t))=v_{x x}(x, t)+f\left(v_{x}(x, t)\right) .
\end{aligned}
$$

The estimate (2.4), the fact that $\psi$ is decreasing, and the definition of $z$ together imply

$$
\begin{aligned}
z_{t}(x, t) & -z_{x x}(x, t)-f\left(z_{x}(x, t)\right) \\
& =\frac{k}{T} \psi-k \psi^{\prime}-f(k \psi)=\frac{k}{T} \psi+k f(\psi)-f(k \psi) \\
& <k \psi[g(k \psi)-g(\psi)]+k f(\psi)-f(k \psi)=0 .
\end{aligned}
$$

(Here we have abbreviated $\psi(x+t / T-1)$ as $\psi$ and have used (2.4) with $s=k \psi$.)

Since (2.6) implies that $z_{x}\left(x_{0}, t_{0}\right)=v_{x}\left(x_{0}, t_{0}\right)$, we can use (2.8) and (2.9) to see that at $\left(x_{0}, t_{0}\right)$

$w_{t}=-w+e^{-t}\left(z_{t}-v_{t}\right)<e^{-t}\left[z_{x x}+f\left(z_{x}\right)-v_{x x}-f\left(v_{x}\right)\right]=e^{-t}\left(z_{x x}-v_{x x}\right)=w_{x x}$,

which contradicts (2.5) and (2.7). This contradiction implies that $\mathcal{T}\left(u_{0}, f\right)$ is finite (and, in particular, is no larger than $T$ ).

\section{BLOWUP FOR VANISHINGLY SMALL MASS}

Given a differentiable, real-valued function $u$ of a single real variable, let $\mathcal{Q}(u, f)$ be given by the formula $\mathcal{Q}(u, f)=u^{\prime}+f(u)$; if $u$ is a function of two variables $x$ and $t$, let $\mathcal{Q}(u, f)=u_{x}+f(u)$. In either case, we call $\mathcal{Q}(u, f)$ the flux of $u$. Notice that if $u=\mathcal{U}\left(u_{0}, f\right)$ and $q=\mathcal{Q}(u, f)$, then $q$ satisfies the initial-boundary value problem

$$
\begin{cases}q_{t}=q_{x x}+p(x, t) q_{x}, & (x, t) \in(0,1) \times\left(0, \mathcal{T}\left(u_{0}, f\right)\right), \\ q=0, & (x, t) \in\{0,1\} \times\left(0, \mathcal{T}\left(u_{0}, f\right)\right), \\ q(\cdot, 0)=q_{0}:=\mathcal{Q}\left(u_{0}, f\right), & x \in[0,1],\end{cases}
$$

where $p(x, t):=f^{\prime}(u(x, t))$, so the maximum principle guarantees that

$$
\min \left\{q_{0}(s) \mid s \in[0,1]\right\} \leq q(x, t) \leq \max \left\{q_{0}(s) \mid s \in[0,1]\right\}
$$

for all $x$ and $t$. This boundedness of $q$ in terms of the flux of $u_{0}$ provides an important control on $\mathcal{U}\left(u_{0}, f\right)$. The following lemma states conditions under which a function exists that satisfies certain boundary conditions at the endpoints of an interval and that has flux bounded by the flux at those endpoints.

Lemma 3.1. Let $u_{1}: \mathcal{I}_{1} \rightarrow[0, \infty)$ and $u_{2}: \mathcal{I}_{2} \rightarrow[0, \infty)$ have domains that are intervals with a point $c$ in common, for which $u_{1}(c)=u_{2}(c)$. Furthermore, assume that $u_{1}$ has constant nonpositive flux $\bar{q}$ and that $u_{2} \equiv \beta$ is constant (and therefore has constant flux $f(\beta)$ ). If $a \in \mathcal{I}_{1}$ and $b \in \mathcal{I}_{2}$ are the endpoints of a closed interval $\mathcal{I}_{3}$ that has $c$ in its interior, then there is a $C^{3}$ function $u_{3}: \mathcal{I}_{3} \rightarrow[0, \infty)$ such that

1. $u_{3}(a)=u_{1}(a), u_{3}^{\prime}(a)=u_{1}^{\prime}(a), u_{3}^{\prime \prime}(a)=u_{1}^{\prime \prime}(a)$, and $u_{3}^{\prime \prime \prime}(a)=u_{1}^{\prime \prime \prime}(a)$;

2. $u_{3}(b)=u_{2}(b), u_{3}^{\prime}(b)=u_{2}^{\prime}(b), u_{3}^{\prime \prime}(b)=u_{2}^{\prime \prime}(b)$, and $u_{3}^{\prime \prime \prime}(b)=u_{2}^{\prime \prime \prime}(b)$;

3. $u^{\prime} \leq 0$ on $\mathcal{I}_{3}$

4. $\bar{q} \leq \mathcal{Q}\left(u_{3}, f\right) \leq f(\beta)$ on $\mathcal{I}_{3}$. 
Proof. We present a proof in the case that $a<b$; the opposite case can be handled similarly (or can be reduced to the first case by appropriate transformations).

Set $\alpha:=u_{1}(a)$. Since $u_{1}^{\prime}=\bar{q}-f(u) \leq 0$ and $u_{1}(c)=\beta$, we see that $\alpha \geq \beta$. The only case in which $\alpha=\beta$ is when $u_{1}$ and $u_{2}$ are identically zero, in which case we could take $u_{3} \equiv 0$; setting aside that case, we can assume that $\alpha>\beta$ and that if $\beta=0$, then $\bar{q}<0$, so $f(s)-\bar{q} \geq \rho>0$ for some constant $\rho$ and all $s \in[\beta, \alpha]$.

Suppose there is a $C^{2}$ function $v:(\beta, \alpha] \rightarrow[0, \infty)$ that satisfies the inequality

$$
v(s) \leq \frac{1}{f(s)-f(\beta)}-\frac{1}{f(s)-\bar{q}}
$$

for all $s \in(\beta, \alpha]$ for which $f(s)>f(\beta)$, that satisfies the integral condition

$$
\int_{\beta}^{\alpha} v(s) d s=b-c
$$

and that satisfies the boundary conditions

$$
\lim _{s \downarrow \beta} \frac{1}{v(s)}=\lim _{s \downarrow \beta} \frac{v^{\prime}(s)}{[v(s)]^{3}}=\lim _{s \downarrow \beta} \frac{v(s) v^{\prime \prime}(s)-3\left[v^{\prime}(s)\right]^{2}}{[v(s)]^{5}}=v(\alpha)=v^{\prime}(\alpha)=v^{\prime \prime}(\alpha)=0 .
$$

If such a $v$ exists, then it is straightforward, if a bit tedious, to check that defining $w:[\beta, \alpha] \rightarrow[a, b]$ by the formula

$$
w(\sigma)=b-\int_{\beta}^{\sigma}\left[v(s)+\frac{1}{f(s)-\bar{q}}\right] d s,
$$

and then defining $u_{3}$ to be $w^{-1}$ will result in $u_{3}$ having the desired properties.

Since $f^{\prime}(\beta)$ exists, there are constants $k, \varepsilon_{1}>0$ and a function $h:(\beta, \alpha] \rightarrow[0, \infty)$ such that

$$
h(s) \geq \frac{k}{s-\beta}
$$

for all $s \in\left(\beta, \beta+\varepsilon_{1}\right]$ and

$$
h(s) \leq \frac{1}{f(s)-f(\beta)}-\frac{1}{f(s)-\bar{q}}
$$

for all $s \in(\beta, \alpha]$ for which $f(s)>f(\beta)$. Pick $\varepsilon_{2}$ such that

$$
0<\varepsilon_{2}<\min \left\{\varepsilon_{1}, k^{4},\left(\frac{b-c}{4}\right)^{4}, \varepsilon_{1} \exp \left[\frac{c-b}{k}\right]\right\}
$$

and define $v(s):=(s-\beta)^{-3 / 4}$ for $s \in\left(\beta, \beta+\varepsilon_{2}\right]$. It is easy to see that $v$ satisfies the necessary boundary conditions at $\beta$. Since $\varepsilon_{2}<\min \left\{\varepsilon_{1}, k^{4}\right\}, v \leq h$ on $\left(\beta, \beta+\varepsilon_{2}\right]$. Since

$$
\begin{aligned}
& \varepsilon_{2}<\min \left\{\left(\frac{b-c}{4}\right)^{4}, \varepsilon_{1} \exp \left[\frac{c-b}{k}\right]\right\}, \\
& \int_{\beta}^{\beta+\varepsilon_{2}} v(s) d s<b-c<\int_{\beta+\varepsilon_{2}}^{\beta+\varepsilon_{1}} h(s) d s .
\end{aligned}
$$

Thus, $v$ can be extended to the entire interval $(\beta, \alpha]$ in such a way that it is $C^{2}$, is nonnegative, is bounded above by $h$, and satisfies the boundary condition at $\alpha$ and the integral condition (3.1). 
Proof of Theorem 1.2, Let $\varepsilon>0$ be given. Assume without loss of generality that $\varepsilon<1$. Let $\psi$ be the function given in Lemma 2.1.

Assuming (SQ) holds, we can pick $N>1$ so large that

$$
\int_{N}^{\infty} \frac{d s}{g(s)}<\frac{\varepsilon}{4}
$$

Pick $k>0$ so large that

$$
\frac{N}{k}+\frac{\varepsilon}{4 N}<1
$$

and

$$
\frac{N^{2}}{2 k}<\frac{\varepsilon}{4}
$$

Assuming (SL4) holds, we can pick $\lambda \in(0,1)$ such that

$$
\liminf _{s \uparrow \infty}[g(s)-g(\lambda s)]>0 .
$$

Assuming (SL1) holds, (3.2) and the continuity of $g$ imply that we can pick $\mu>0$ so small that

$$
g(s)-g(\lambda s) \geq \mu
$$

for all $s \geq \psi(1) / \lambda$. Pick $c_{2}>0$ so small that $\psi\left(c_{2}\right)>N$,

$$
c_{2}<1-\left[\frac{N}{k}+\frac{\varepsilon}{4 N}\right]
$$

and

$$
\int_{0}^{c_{2}} \psi(s) d s \leq \lambda\left[\frac{\varepsilon}{2}-\frac{k c_{2}}{\mu}\right] .
$$

Calculating, we find that

$$
\begin{gathered}
c_{2} \psi\left(c_{2}\right) \leq \int_{0}^{c_{2}} \psi(x) d x=\int_{\psi\left(c_{2}\right)}^{\infty} \frac{d s}{g(s)}<\int_{N}^{\infty} \frac{d s}{g(s)}<\frac{\varepsilon}{4}, \\
c_{2}+\int_{0}^{\psi\left(c_{2}\right)} \frac{d s}{k+f(s)} \leq c_{2}+\int_{0}^{N} \frac{d s}{k}+\frac{1}{N} \int_{N}^{\infty} \frac{d s}{g(s)}<c_{2}+\frac{N}{k}+\frac{\varepsilon}{4 N}<1,
\end{gathered}
$$

and

$$
\int_{0}^{\psi\left(c_{2}\right)} \frac{s d s}{k+f(s)} \leq \int_{0}^{N} \frac{s d s}{k}+\int_{N}^{\infty} \frac{d s}{g(s)}<\frac{N^{2}}{2 k}+\frac{\varepsilon}{4}<\frac{\varepsilon}{2} .
$$

Thus, the continuity of $\psi$ implies that we can pick $c_{0} \in\left(0, c_{2}\right)$ so small that

$$
\begin{aligned}
& c_{2} \psi\left(c_{2}-c_{0}\right)<\frac{\varepsilon}{2}, \\
& c_{2}+\int_{0}^{\psi\left(c_{2}-c_{0}\right)} \frac{d s}{k+f(s)}<1,
\end{aligned}
$$

and

$$
\int_{0}^{\psi\left(c_{2}-c_{0}\right)} \frac{s d s}{k+f(s)}<\frac{\varepsilon}{2}
$$


By (3.5) and (3.6), we can pick $c_{3} \in\left(c_{2}, 1\right)$ so small that

$$
c_{4}:=c_{3}+\int_{0}^{\psi\left(c_{2}-c_{0}\right)} \frac{d s}{k+f(s)}<1
$$

and

$$
\left(c_{3}-c_{2}\right) \psi\left(c_{2}-c_{0}\right)+\int_{0}^{\psi\left(c_{2}-c_{0}\right)} \frac{s d s}{k+f(s)}<\frac{\varepsilon}{2} .
$$

Let $h:\left(c_{0}, c_{2}\right) \rightarrow \mathbb{R}$ be defined by the formula

$$
h(\sigma)=\sigma \psi\left(\sigma-c_{0}\right)+\int_{\psi\left(c_{2}-c_{0}\right)}^{\psi\left(\sigma-c_{0}\right)} \frac{d s}{g(s)} .
$$

Note that $h$ is continuous and satisfies $\lim _{\sigma \downarrow c_{0}} h(\sigma)=\infty$ and, by (3.4),

$$
\lim _{\sigma \uparrow c_{2}} h(\sigma)=c_{2} \psi\left(c_{2}-c_{0}\right)<\frac{\varepsilon}{2}
$$

so the Intermediate Value Theorem implies that we can pick $c_{1} \in\left(c_{0}, c_{2}\right)$ such that $h\left(c_{1}\right)=\varepsilon / 2$.

Define $j:\left[0, \psi\left(c_{2}-c_{0}\right)\right] \rightarrow\left[c_{3}, c_{4}\right]$ by the formula

$$
j(\sigma)=c_{3}+\int_{\sigma}^{\psi\left(c_{2}-c_{0}\right)} \frac{d s}{k+f(s)} .
$$

Since $j^{\prime}(\sigma)=-1 /(k+f(\sigma))<0, j(0)=c_{4}$, and $j\left(\psi\left(c_{2}-c_{0}\right)\right)=c_{3}, j$ is a bijection. Let $y=j^{-1}$, and note that $y^{\prime}(x)=1 / j^{\prime}(y(x))=-(k+f(y(x)))$, so $\mathcal{Q}(y, f)=-k$.

Define $w_{0}:[0,1] \rightarrow[0, \infty)$ by the formula

$$
w_{0}(x)= \begin{cases}\psi\left(c_{1}-c_{0}\right) & \text { if } x \in\left[0, c_{1}\right], \\ \psi\left(x-c_{0}\right) & \text { if } x \in\left(c_{1}, c_{2}\right], \\ \psi\left(c_{2}-c_{0}\right) & \text { if } x \in\left(c_{2}, c_{3}\right], \\ y(x) & \text { if } x \in\left(c_{3}, c_{4}\right], \\ 0 & \text { if } x \in\left(c_{4}, 1\right],\end{cases}
$$

and observe that $w_{0}$ is continuous. Note that

$$
\begin{aligned}
\int_{0}^{c_{2}} w_{0}(x) d x & =c_{1} \psi\left(c_{1}-c_{0}\right)+\int_{c_{1}}^{c_{2}} \psi\left(x-c_{0}\right) d x \\
& =c_{1} \psi\left(c_{1}-c_{0}\right)+\int_{\psi\left(c_{2}-c_{0}\right)}^{\psi\left(c_{1}-c_{0}\right)} \frac{d s}{g(s)}=h\left(c_{1}\right)=\frac{\varepsilon}{2},
\end{aligned}
$$

and

$$
\begin{aligned}
\int_{c_{2}}^{1} w_{0}(x) d x & =\int_{c_{2}}^{c_{3}} \psi\left(c_{2}-c_{0}\right) d x+\int_{c_{3}}^{c_{4}} y(x) d x \\
& =\left(c_{3}-c_{2}\right) \psi\left(c_{2}-c_{0}\right)+\int_{0}^{\psi\left(c_{2}-c_{0}\right)} \frac{s d s}{k+f(s)}<\frac{\varepsilon}{2},
\end{aligned}
$$

so, in particular,

$$
\int_{0}^{1} w_{0}(x) d x<\varepsilon
$$

Now, the graph of $w_{0}$ consists of horizontal line segments alternating with curves of constant nonpositive flux, and $\mathcal{Q}\left(w_{0}, f\right) \geq-k$ wherever it's defined. Therefore, 


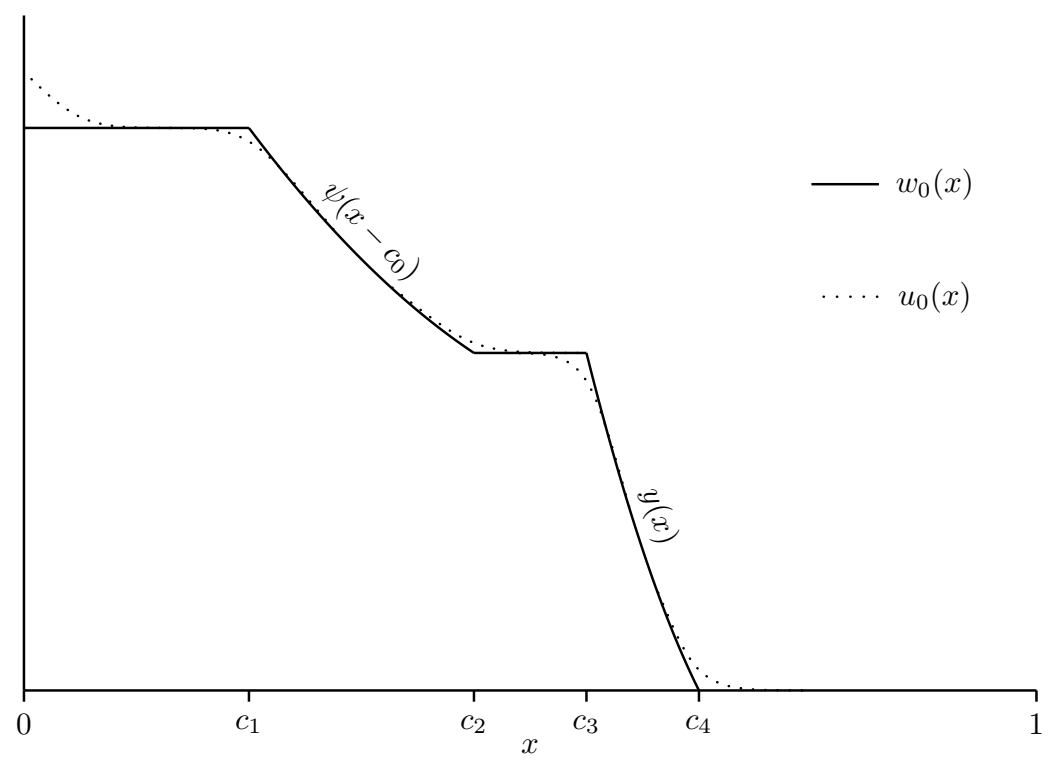

FIgURE 1. $w_{0}$ and $u_{0}$

by Lemma 3.1 it is possible to get a $C^{3}$ function $u_{0}:[0,1] \rightarrow[0, \infty)$ by smoothing out the corners in the graph of $w_{0}$ in such a way that $\mathcal{Q}\left(u_{0}, f\right)(x) \geq-k$ on $[0,1]$, $\mathcal{Q}\left(u_{0}, f\right)(x)=0$ for $x \in\{0,1\}, \mathcal{M}\left(u_{0}\right)<\varepsilon$, and

$$
\int_{0}^{c_{2}} u_{0}(x) d x \geq \frac{\varepsilon}{2} .
$$

(See Figure 1) We shall show that $\mathcal{T}\left(u_{0}, f\right) \leq T:=c_{2} / \mu$.

Suppose, to the contrary, that $\mathcal{T}\left(u_{0}, f\right)>T$. Let $u=\mathcal{U}\left(u_{0}, f\right)$ and $q=\mathcal{Q}(u, f)$. As we noted previously, the maximum principle implies that $q(x, t) \geq-k$ for all $(x, t) \in[0,1] \times\left[0, \mathcal{T}\left(u_{0}, f\right)\right)$, since $\mathcal{Q}\left(u_{0}, f\right) \geq-k$. In particular, $q\left(c_{2}, t\right) \geq-k$.

Let

$$
\Omega=\left\{(x, t) \in\left(0, c_{2}\right) \times(0, T) \mid x>c_{2}\left(1-\frac{t}{T}\right)\right\} .
$$

Define $v, z$, and $w$ on $\bar{\Omega}$ by the formulas

$$
\begin{gathered}
v(x, t)=\int_{0}^{x} u(s, t) d s \\
z(x, t)=\frac{1}{\lambda} \int_{0}^{x-c_{2}(1-t / T)} \psi(s) d s,
\end{gathered}
$$

and

$$
w(x, t)=e^{-t}(z(x, t)-v(x, t)) .
$$

As in the proof of Theorem 1.1 we can show that $w>0$ somewhere in $\bar{\Omega}$ but attains a positive maximum nowhere on that compact set, which will yield a contradiction.

That $w>0$ somewhere in $\bar{\Omega}$ follows from the fact that $w(0, T)=0$ and $w_{x}(x, T) \rightarrow \infty$ as $x \downarrow 0$. It is easy to see that $w \leq 0$ along the boundary segment $x=c_{2}(1-t / T)$. Along the boundary segment $x=c_{2}$, the lower bound on $q$ 
gives

$$
v_{t}\left(c_{2}, t\right)=\int_{0}^{c_{2}} u_{t}(s, t) d s=u_{x}\left(c_{2}, t\right)+f\left(u\left(c_{2}, t\right)\right)=q\left(c_{2}, t\right) \geq-k
$$

SO

$$
v\left(c_{2}, t\right) \geq-k t+v\left(c_{2}, 0\right) \geq-k T+\int_{0}^{c_{2}} u_{0}(s) d s \geq \frac{\varepsilon}{2}-k T
$$

by (3.7). On the other hand,

$$
z\left(c_{2}, t\right)=\frac{1}{\lambda} \int_{0}^{c_{2} t / T} \psi(s) d s \leq \frac{1}{\lambda} \int_{0}^{c_{2}} \psi(s) d s \leq \frac{\varepsilon}{2}-\frac{k c_{2}}{\mu}
$$

by $(3.3)$, so $w\left(c_{2}, t\right) \leq 0$.

As in the proof of Theorem 1.1. $v_{t}=v_{x x}+f\left(v_{x}\right)$, and

$$
\begin{aligned}
z_{t}-\left[z_{x x}+f\left(z_{x}\right)\right] & =\frac{c_{2}}{\lambda T} \psi-\frac{1}{\lambda} \psi^{\prime}-f(\psi / \lambda)=\frac{c_{2}}{\lambda T} \psi+\frac{1}{\lambda} f(\psi)-f(\psi / \lambda) \\
& =\frac{\psi}{\lambda}\left[\frac{c_{2}}{T}+g(\psi)-g(\psi / \lambda)\right] \leq \frac{\psi}{\lambda}\left[\frac{c_{2}}{T}-\mu\right]=0
\end{aligned}
$$

so there will be nowhere that a positive maximum could be attained.

\section{REFERENCES}

1. Nicholas D. Alikakos, Peter W. Bates, and Christopher P. Grant, Blow up for a diffusionadvection equation, Proc. Roy. Soc. Edinburgh Sect. A 113 (1989), 181-190. MR 91e:35029

2. Miguel Escobedo, Juan Luis Vázquez, and Enrike Zuazua, Asymptotic behaviour and sourcetype solutions for a diffusion-convection equation, Arch. Rational Mech. Anal. 124 (1993), no. 1, 43-65. MR 94j:35077

3. - A diffusion-convection equation in several space dimensions, Indiana Univ. Math. J. 42 (1993), no. 4, 1413-1440. MR 95e:35090

4. Miguel Escobedo and Enrike Zuazua, Large time behavior for convection-diffusion equations in $\mathbf{R}^{n}$, J. Funct. Anal. 100 (1991), 119-161. MR 92i:35063

5. Miguel Escobedo and Enrique Zuazua, Long-time behavior for a convection-diffusion equation in higher dimensions, SIAM J. Math. Anal. 28 (1997), no. 3, 570-594. MR 97m:35120

6. Philip Hartman, Ordinary differential equations, Birkhäuser, Boston, 1982. MR 83e:34002

7. O. A. Ladyzenskaja, V. A. Solonnikov, and N. N. Ural'ceva, Linear and quasilinear equations of parabolic type, Translations of Mathematical Monographs, vol. 23, American Mathematical Society, Providence, 1967. MR 39:3159b

8. Murray H. Protter and Hans F. Weinberger, Maximum principles in differential equations, Prentice-Hall, Englewood Cliffs, NJ, 1967. MR 36:2935

9. Enrique Zuazua, Weakly nonlinear large time behavior in scalar convection-diffusion equations, Differential Integral Equations 6 (1993), no. 6, 1481-1491. MR 94j:35084

Department of Mathematics, Northwestern University, 2033 Sheridan Road, EvansTON, ILLINOIS 60208-2730

E-mail address: tfisher@math.nwu.edu

$U R L: \mathrm{http} / /$ www .math.nwu.edu/graduate/homepages/todd.fisher.html

Department of Mathematics, Brigham Young University, Provo, Utah 84602

E-mail address: grant@math.byu.edu

URL: http://www.math.byu.edu/ grant 